\title{
A Stokes-Brinkman model of the fluid flow in a periodic cell with a porous body using the boundary element method
}

\author{
R.F. Mardanov ${ }^{\mathrm{a}}$, S.K. Zaripov ${ }^{\mathrm{a}}$, V.F. Sharafutdinov ${ }^{\mathrm{a}}$, S.J.Dunnett ${ }^{\mathrm{b}}$ \\ ${ }^{a}$ Kazan Federal University, Kazan, Russia \\ ${ }^{b}$ Loughborough University, Loughborough, UK
}

\begin{abstract}
The problem of viscous incompressible flow in a periodic cell with a porous body is solved. The Stokes flow model is adopted to describe the flow outside the body and the Brinkman equation is applied to find the filtration velocity field inside the porous domain. The conditions on the boundary between the free fluid and the porous medium for the porous body of arbitrary shape are obtained. The boundary value problem for the joint solution of the biharmonic and Brinkman equations for the stream functions outside and inside the porous body are then solved using a boundary element method. Good agreement of the numerical and analytical models for the Kuwabara circular cell model is shown for the fluid flow through a porous circular cylinder. The fluid flow past a circular, square, triangular cylinders and a circular body of uneven surface (an idealized model of a viral capsid) in a rectangular periodic cell are calculated. Comparison of the results obtained with the numerical solution from a CFD ANSYS/FLUENT model shows good accuracy of the developed mathematical model.
\end{abstract}

Keywords: porous body, Stokes flow, Brinkman equation, boundary element method

Email addresses: Renat.Mardanov@kpfu.ru (R.F. Mardanov), shamil.zaripov@kpfu.ru (S.K. Zaripov)

Preprint submitted to Journal of ${ }^{A} T_{E} X$ Templates

December 19, 2017 


\section{Nomenclature}

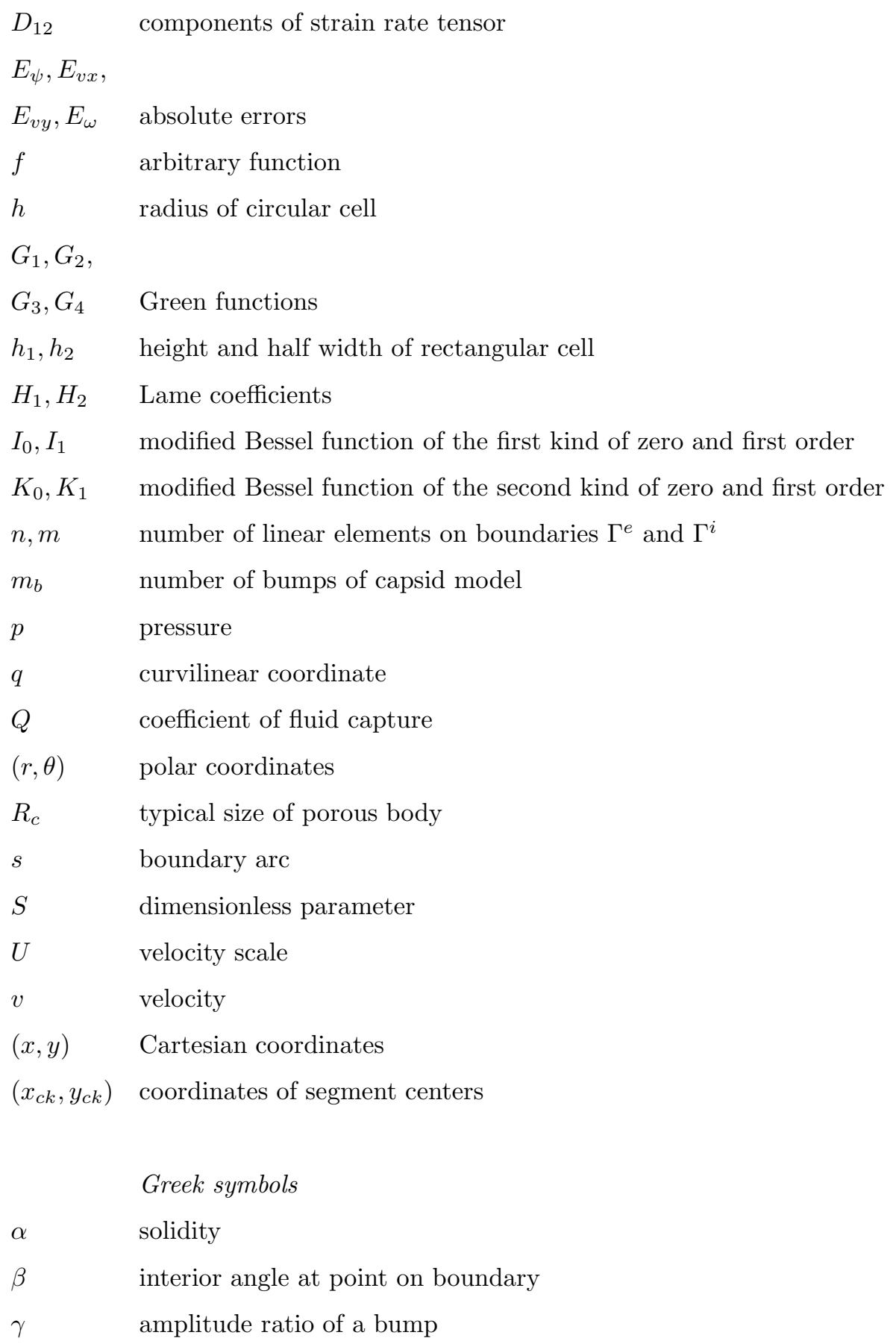




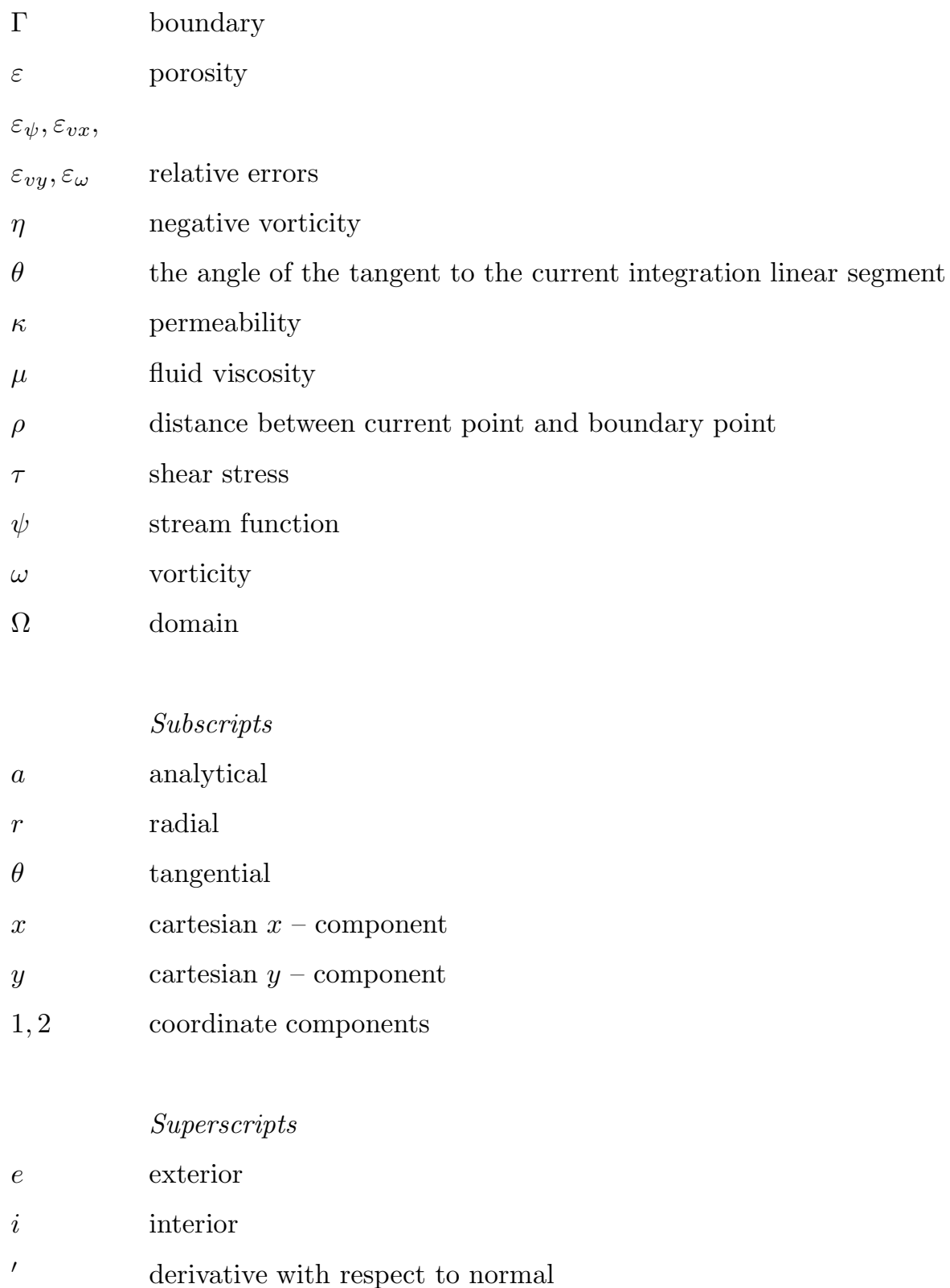




\section{Introduction}

The solution of the problem of fluid flow through porous bodies is used to describe many hydrodynamical processes in environmental and medical science.

5 For example, such flows are found in aerosol filters and respirators and for particle shaped viruses in biofluids. In the case of aerosol filters porous bodies can be used as elements to increase the efficiency of the deposition of aerosol particles [1, 2]. In order to calculate the two-phase flows of dusty air for such filters it is important to develop efficient mathematical models of fluid flow past porous bodies in periodic cells [3].

The fluid flow through a circular porous cylinder assuming potential flow of an incompressible fluid was modelled in work [4].

One of the problems with modelling fluid flow in domains containing porous medium is connected with the formulation of the boundary conditions on the interface between the free space and porous medium. The choice of the conditions depends on the mathematical model adopted. Various boundary conditions are studied in the works of Beavers and Joseph [5], Saffman [6], Neale and Nader [7], Haber and Mauri [8], Vafai and Thiyagaraja 9], Sahraoui and Kaviany [10], Ochoa-Tapia and Whitaker [11, 12].

The analytical solution of the problem of the fluid flow past an isolated porous cylinder and a system of porous cylinders using a cell model was firstly obtained by Stechkina [13]. The cell model used was based on the widely adopted Kuwabara cell model [14] and included the Stokes flow model [15] outside and the Brinkman equation [16] inside the porous cylinder. The cell 25 model with Kuwabara boundary conditions was also used by Deo et al. [17] and Kirsh [1] to determine the velocity field of the flow over and through a porous cylinder in the case of small Reynolds number flow using the analytical solution and the collocation method.

A review of analytical investigations of fluid flow past porous cylinders and 30 spheres is given in the works of Deo et al. [18] and Vasin and Filippov [19]. Generally, the cell model is an approximate model of fluid flow and its accuracy 
depends on the porosity of the porous medium. To obtain an accurate fluid flow velocity field numerical models using the real array geometry should be adopted.

Viscous flow models for flows through porous bodies usually adopt the combination of the Stokes model in the free space and the Darcy or Brinkman model in the porous medium. Such models were used to study the viscous flow through isolated porous cylinders and spheres by Masliyah and Polikar [20], Nandakumar and Masliyah 21], Noymer et al [22], Vanni [23], Vainshtein et al [24, 25].

The Navier-Stokes equations with Darcy and Forchmeier terms were solved numerically to simulate the fluid flow past a porous cylinder in Beckermann and Viskanta [26], Vafai and Kim [27], Basu and Khalili [28], Bhattacharyya et al [29].

Using the Boundary Element Method (BEM) for solving the problem of fluid flow through porous bodies has advantages compared with the finite volume (FVM) or finite differences (FDM) methods due to its reduction of the dimension of the boundary value problem. Additionally adopting the BEM enables boundary value problems to be solved for porous bodies of any shape. Whilst the FVM and FDM have difficulties with the numerical solution of the fluid flow through porous bodies of complex shape. The majority of previous work in the area is devoted to the study of fluid flow through circular or rectangular bodies [3, 30]. But there are applications where it is necessary to study the fluid flow through porous bodies of arbitrary shape (for example, the flow through aerosol fibrous filters containing deposit or the fluid through a viral capsid). Presently these problems remains unsolved. In this paper a mathematical model of fluid flow through arbitrary shaped porous bodies is developed using the BEM approach.

The fluid flow past a periodic row past of arbitrary shaped porous bodies, 60 is considered under the assumption of viscous incompressible flow. The approximate periodic circular [14] and rectangular cell models are used to formulate the fluid flow problem. The Stokes flow model is adopted outside 
the body in the cell and the Brinkman model inside the porous body to describe the flow velocity. The resulting boundary value problem for the stream function in the two domains with boundary conditions on the interface between the free space and the porous medium is formulated. The boundary conditions include the stream function and vorticity as variables and can be used for arbitrary curvilinear boundaries. The boundary element method (BEM) is then used to solve the boundary value problems. The numerical solution obtained is compared with the analytical solution in the circular cell case and good agreement of the two solutions is shown. The fluid flow past circular, square, triangular cylinders and a circular body with an uneven surface (an idealized model of a viral capsid) in a rectangular periodic cell is also calculated. Comparison of the numerical results with corresponding 75 data obtained using CFD ANSYS/FLUENT (www.ansys.com) shows good correlation of the developed BEM model and FVM solution.

\section{The problem statement}

The two-dimensional flow of an incompressible fluid with speed $U$ in a periodic cell with a porous body at a small Reynolds numbers is considered.

80 The permeability $\kappa$ of the porous medium is assumed to be constant. Due to the fluid flow symmetry we select as a calculation domain the upper part of the periodic cell that consists of $\Omega=\Omega^{e} \cup \Omega^{i}$ where $\Omega^{e}$ is the free fluid space and $\Omega^{i}$ the porous body domain (Fig. 1). The line $A D$ is the symmetry axis. All quantities in the domains $\Omega^{e}$ and $\Omega^{i}$ are denoted by indexes $e$ and $i$.

85 The fluid flow in the domain $\Omega^{e}$ limited by the boundary $\Gamma^{e}$ is described by the Stokes model given by equation (1)

$$
\nabla p^{e}=-\mu^{e} \operatorname{rot} \omega^{e}
$$

where $p^{e}$ is the pressure of outer flow, $\mu^{e}$ is the fluid viscosity, $\boldsymbol{\omega}^{e}=\left(0,0, \omega^{e}\right)$ is the flow vorticity vector. The fluid flow in the porous domain $\Omega^{i}$ limited by 


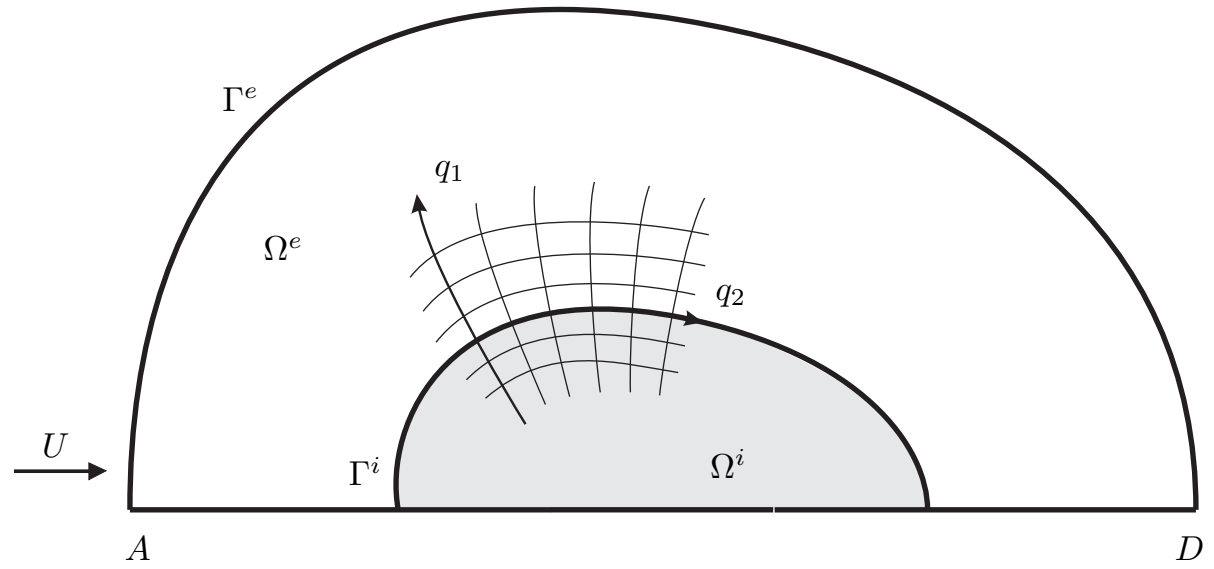

Figure 1: Fluid flow domain

the boundary $\Gamma^{i}$ is described by the Brinkman model

$$
\nabla p^{i}=-\frac{\mu^{e}}{\kappa} \boldsymbol{v}^{i}-\mu^{i} \operatorname{rot} \boldsymbol{\omega}^{i}
$$

90

where $p^{i}$ is the pressure, $\boldsymbol{\omega}^{i}=\left(0,0, \omega^{i}\right)$ is the vorticity, $\boldsymbol{v}^{i}$ is the average filtration velocity and $\mu^{i}$ is the fluid viscosity in the porous domain. The fluid viscosity in the free space and the porous body $\mu^{e}$ and $\mu^{i}$ differs due to the additional fluid drag in the porous medium.

Considering the curvilinear coordinate system $\left(q_{1}, q_{2}\right)$ (Fig. 1). For convenience the coordinate system is selected such that on the boundary $\Gamma=\Gamma^{e} \bigcap \Gamma^{i}$ between the free space and the porous medium condition (3) is satisfied

$$
q_{1} \equiv q_{1}^{0}=\text { const } .
$$

The continuity equations for both domains are written in the form

$$
\frac{\partial\left(H_{2} v_{1}^{e}\right)}{\partial q_{1}}+\frac{\partial\left(H_{1} v_{2}^{e}\right)}{\partial q_{2}}=0, \quad \frac{\partial\left(H_{2} v_{1}^{i}\right)}{\partial q_{1}}+\frac{\partial\left(H_{1} v_{2}^{i}\right)}{\partial q_{2}}=0
$$

where $H_{1}, H_{2}$ are the Lame coefficients for the curvilinear coordinate system, $v_{1}^{e}, v_{2}^{e}, v_{1}^{i}, v_{2}^{i}$ are the projections of the velocity vectors on the coordinate axes $q_{1}$ and $q_{2}$. The form of equations (4) enables the introduction of the stream 
functions $\psi^{e}$ and $\psi^{i}$ as

$$
H_{2} v_{1}^{e}=\frac{\partial \psi^{e}}{\partial q_{2}}, \quad H_{1} v_{2}^{e}=-\frac{\partial \psi^{e}}{\partial q_{1}}, \quad H_{2} v_{1}^{i}=\frac{\partial \psi^{i}}{\partial q_{2}}, \quad H_{1} v_{2}^{i}=-\frac{\partial \psi^{i}}{\partial q_{1}} .
$$

The components $\omega^{e}$ and $\omega^{i}$ of the vorticity vectors in the coordinate system considered are written in the form

$$
\omega^{e}=\frac{1}{H_{1} H_{2}}\left(\frac{\partial\left(H_{2} v_{2}^{e}\right)}{\partial q_{1}}-\frac{\partial\left(H_{1} v_{1}^{e}\right)}{\partial q_{2}}\right), \quad \omega^{i}=\frac{1}{H_{1} H_{2}}\left(\frac{\partial\left(H_{2} v_{2}^{i}\right)}{\partial q_{1}}-\frac{\partial\left(H_{1} v_{1}^{i}\right)}{\partial q_{2}}\right) .
$$

$$
\frac{1}{H_{1}} \frac{\partial p^{i}}{\partial q_{1}}=-\frac{\mu^{e}}{\kappa} v_{1}^{i}-\mu^{i} \frac{1}{H_{2}} \frac{\partial \omega^{i}}{\partial q_{2}}, \quad \frac{1}{H_{2}} \frac{\partial p^{i}}{\partial q_{2}}=-\frac{\mu^{e}}{\kappa} v_{2}^{i}+\mu^{i} \frac{1}{H_{1}} \frac{\partial \omega^{i}}{\partial q_{1}} .
$$

Eliminating the pressure in (9) and (10) and using (7) the fluid flow equations can be written in the form that includes the stream functions $\psi^{e}$ and $\psi^{i}$

$$
\begin{gathered}
\Delta^{2} \psi^{e}=0, \\
\Delta^{2} \psi^{i}-\frac{1}{\kappa} \Delta \psi^{i}=0 .
\end{gathered}
$$

The conditions on the boundary $\Gamma^{e}$ depend on the periodic cell model adopted [17]. The symmetry conditions are taken on the line $A D$.

To complete the boundary value problem for the fluid flow equations (11), (12) that are partial differential equations of fourth order it is necessary to take four conditions on the interface $\Gamma^{i}$ between the free space and the porous 
medium. The condition of velocity equality $\boldsymbol{v}^{e}=\boldsymbol{v}^{i}$ on the boundary can be written in the form

$$
v_{1}^{e}=v_{1}^{i}, \quad v_{2}^{e}=v_{2}^{i} .
$$

Taking into account (5) the relations (13) can be rewritten as

$$
\psi^{e}=\psi^{i}, \quad \frac{\partial \psi^{e}}{\partial q_{1}}=\frac{\partial \psi^{i}}{\partial q_{1}} .
$$

From (3) it follows that the condition of pressure equality on the boundary $p^{e}=p^{i}$ can be presented in the form

$$
\frac{\partial p^{e}}{\partial q_{2}}=\frac{\partial p^{i}}{\partial q_{2}} .
$$

Taking into account (5), (9) and (10) the last relation can be written in the form

$$
\frac{\partial \omega^{e}}{\partial q_{1}}=\frac{\partial}{\partial q_{1}}\left(\frac{1}{\kappa} \psi^{i}+\frac{\mu^{i}}{\mu^{e}} \omega^{i}\right) .
$$

The shear stresses equality $\tau^{e}=\tau^{i}$ [17] can be written as

$$
2 \mu^{e} D_{12}^{e}=2 \mu^{i} D_{12}^{i}
$$

The components $D_{12}^{e}$ and $D_{12}^{i}$ of the strain rate tensor in the curvilinear coordinate system are written as:

$$
\begin{aligned}
& 2 D_{12}^{e}=\frac{H_{1}}{H_{2}} \frac{\partial}{\partial q_{2}}\left(\frac{v_{1}^{e}}{H_{1}}\right)+\frac{H_{2}}{H_{1}} \frac{\partial}{\partial q_{1}}\left(\frac{v_{2}^{e}}{H_{2}}\right), \\
& 2 D_{12}^{i}=\frac{H_{1}}{H_{2}} \frac{\partial}{\partial q_{2}}\left(\frac{v_{1}^{i}}{H_{1}}\right)+\frac{H_{2}}{H_{1}} \frac{\partial}{\partial q_{1}}\left(\frac{v_{2}^{i}}{H_{2}}\right) .
\end{aligned}
$$

Substituting the relationships from (5) in the formulas above and taking into account (6) -(8) gives the equality

$$
2\left(D_{12}^{e}-D_{12}^{i}\right)=\omega^{e}-\omega^{i} .
$$

Using the assumption that $\mu^{e}=\mu^{i}$ [17] the condition of shear stresses equality (17) is written in the form

$$
\omega^{e}=\omega^{i}
$$

From (6), (13), (21) it follows that the velocity and its derivative is continuous at the interface between the free space and the porous medium. 


\section{Statement of the mathematical problem}

The typical size of the porous body, $R_{c}$ and the fluid velocity $U$ are used as length and velocity scales. The equations of the model described above are written in terms of the dimensionless quantities

$$
\tilde{q}=\frac{q}{R_{c}}, \quad \tilde{v}=\frac{v}{U}, \quad \tilde{\psi}=\frac{\psi}{U R_{c}}, \quad \tilde{\omega}=\frac{\omega R_{c}}{U}, \quad \tilde{p}=\frac{p R_{c}}{\mu^{e} U} .
$$

140 the superscript " $\sim$ " has been omitted.

Considering a periodic circular cell with radius $h$ (Fig. 2, a) (Kuwabara model) and a rectangular cell with height $h_{1}$ and width $2 h_{2}$ (Fig. 2, b). Within the Kuwabara model it is possible to obtain an analytical solution of the Stokes-Brinkman problem. The rectangular cell model is a more accurate representation of the fluid flow field but in this case it is only possible to obtain a numerical solution. The solidity $\alpha$ of the periodic cell can be expressed as the ratio of the porous body area to the total cell area. The porosity $\varepsilon$ of the periodic cell is determined as

$$
\varepsilon=1-\alpha
$$

Equation (11) for the stream function $\psi^{e}(x, y)$ in the outer domain $\Omega^{e}$ in dimensionless form is written

$$
\Delta^{2} \psi^{e}=0
$$

The boundary value problem for equation (24) includes the conditions on the boundary $\Gamma^{e}$. In the circular cell case the conditions on the outer boundary $A D$ are

$$
\psi^{e}=y, \quad \omega^{e}=0 .
$$

For the rectangular cell the periodic conditions on the sides $A E$ and $D F$ are given by

$$
\begin{array}{ll}
\psi^{e}\left(h_{2}, y\right)=\psi^{e}\left(-h_{2}, y\right), & \psi^{e^{\prime}}\left(h_{2}, y\right)=-\psi^{e^{\prime}}\left(-h_{2}, y\right), \\
\omega^{e}\left(h_{2}, y\right)=\omega^{e}\left(-h_{2}, y\right), & \omega^{e \prime}\left(h_{2}, y\right)=-\omega^{e \prime}\left(-h_{2}, y\right),
\end{array}
$$




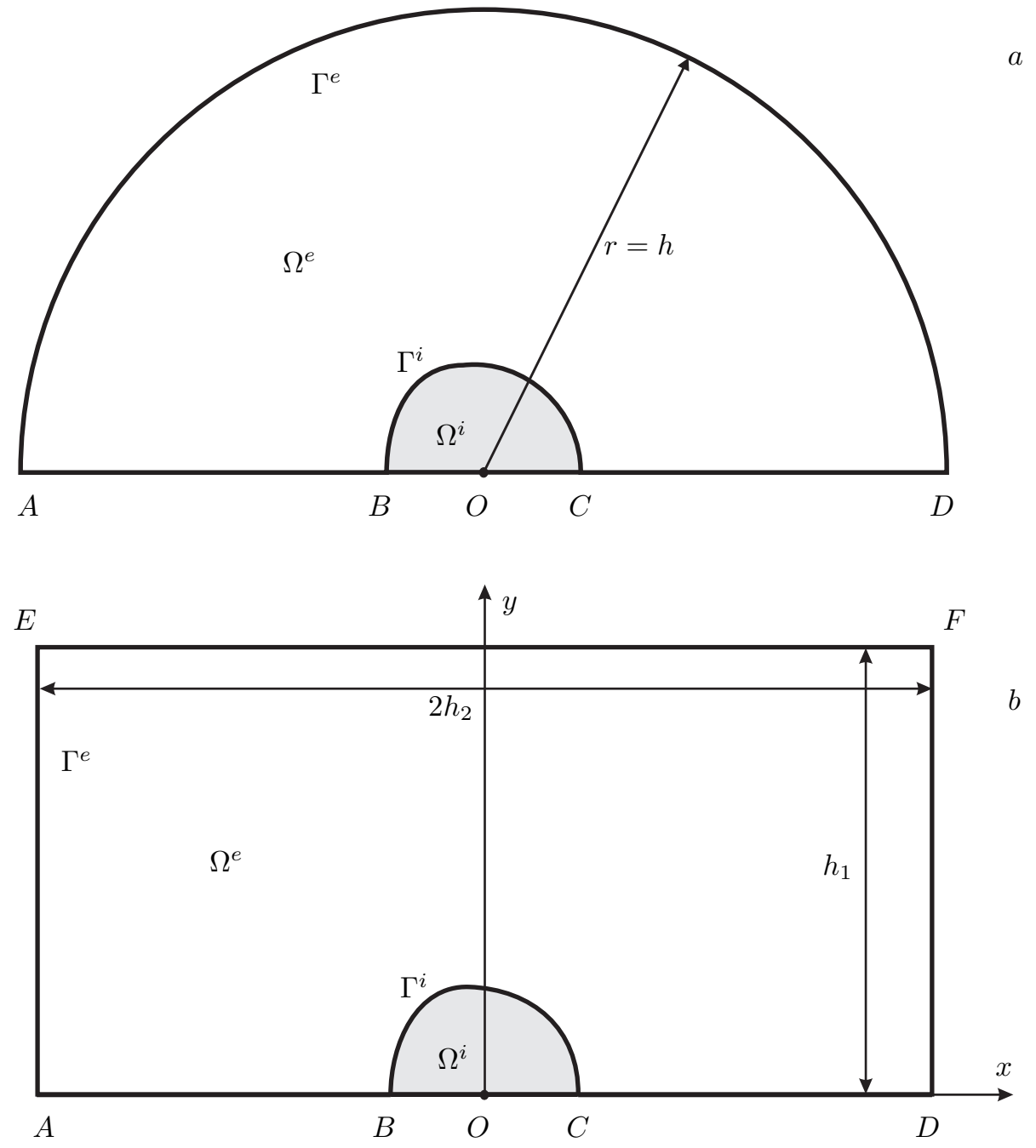

Figure 2: Fluid flow domain for circular $(a)$ and rectangular $(b)$ cells

where prime denotes the derivative with respect to the outer normal $\partial / \partial n$. The symmetry conditions on the line $E F$ are

$$
\psi^{e}=h_{1}, \quad \omega^{e}=0 .
$$

The symmetry conditions on the lines $A B$ and $C D$ are

$$
\psi^{e}=0, \quad \omega^{e}=0 .
$$


In the porous domain $\Omega^{i}$ the flow stream function $\psi^{i}(x, y)$ satisfies equation (12) that follows from the Brinkman equation

$$
\Delta^{2} \psi^{i}-S^{2} \Delta \psi^{i}=0
$$

where $S=R_{c} / \sqrt{\kappa}$ is a dimensionless parameter. The symmetry conditions on the line $B O C$ are

$$
\psi^{i}=0, \quad \omega^{i}=0 .
$$

The boundary conditions (14), (16), (21) on the interface of the free flow

165

$$
\psi^{e}=\psi^{i}, \quad \psi^{e^{\prime}}=-\psi^{i^{\prime}}, \quad-\omega^{e^{\prime}}=S^{2} \psi^{i^{\prime}}+\omega^{i^{\prime}}, \quad \omega^{e}=\omega^{i} .
$$

The introduced curvilinear coordinate system $\left(q_{1}, q_{2}\right)$ is orthogonal. From the relation (3) it follows that the derivative with respect to the coordinate $q_{1}$ on the boundary $\Gamma$ can be replaced by the derivative with respect to the normal to domain boundary. Hence

$$
\frac{1}{H_{1}} \frac{\partial}{\partial q_{1}}=-\frac{\partial}{\partial n}
$$

170 for the domain $\Omega^{e}$ and

$$
\frac{1}{H_{1}} \frac{\partial}{\partial q_{1}}=\frac{\partial}{\partial n}
$$

for the domain $\Omega^{i}$.

The problem is to determine the stream function $\psi(x, y)$, the components of the flow velocity vector $v_{x}(x, y)$ and $v_{y}(x, y)$ and the vorticity $\omega(x, y)$ in the entire flow domain $\Omega$.

\section{Solution}

To solve the boundary value problem (24)-(28), (31) in the domain $\Omega^{e}$ equation (24) of fourth order is written as two equations of second order [3, 31]

$$
\begin{gathered}
\Delta \psi^{e}=\eta^{e} \\
\Delta \eta^{e}=0 .
\end{gathered}
$$


where $\eta^{e}=-\omega^{e}$. The equivalent pair of coupled integral equations 32] is

obtained using the Rayleigh-Green biharmonic boundary formula (see [33]) and Green's second identity,

$$
\begin{aligned}
& \chi(x, y) \psi^{e}(x, y)=\int_{\Gamma^{e}}\left(\psi^{e}(s) G_{1}^{\prime}(x, y, s)-\psi^{e \prime}(s) G_{1}(x, y, s)+\right. \\
& \left.+\eta^{e}(s) G_{2}^{\prime}(x, y, s)-\eta^{e \prime}(s) G_{2}(x, y, s)\right) d s, \\
& \chi(x, y) \eta^{e}(x, y)=\int_{\Gamma^{e}}\left(\eta^{e}(s) G_{1}^{\prime}(x, y, s)-\eta^{e \prime}(s) G_{1}(x, y, s)\right) d s
\end{aligned}
$$

where $\chi(x, y)=2 \pi$ for the interior points $(x, y) \in \Omega^{e}, \chi(x, y)=\beta^{e}$ for the boundary points $(x, y) \in \Gamma^{e}$ ( $\beta^{e}$ is the interior angle at a point on the boundary $\left.\Gamma^{e}\right), s$ is the boundary arc. The corresponding Greens functions are written as

$$
G_{1}=\ln \rho, \quad G_{2}=\frac{\rho^{2}}{4}(\ln \rho-1)
$$

where

$$
\rho(x, y, s)=\sqrt{\left(x_{1}(s)-x\right)^{2}+\left(y_{1}(s)-y\right)^{2}}
$$

and $\left(x_{1}, y_{1}\right)$ is the coordinate of the boundary point with arc abscissa $s$.

The boundary $\Gamma^{e}=\bigcup_{j=1}^{n} \Gamma_{j}^{e}$ is approximated as a number of linear elements $\Gamma_{j}^{e}$ (linear segments). The functions $\psi^{e}(s), \psi^{e^{\prime}}(s), \eta^{e}(s), \eta^{e^{\prime}}(s)$ are approximated by piece-wise constant functions with values $\psi_{j}^{e}, \psi_{j}^{e \prime}, \eta_{j}^{e}, \eta_{j}^{e \prime}$ on the single element $\Gamma_{j}^{e}$. Eq. (36), (37) can be rewritten in the discrete form

$$
\begin{aligned}
\chi(x, y) \psi^{e}(x, y)=\sum_{j=1}^{n}\left\{\psi_{j}^{e} \int_{\Gamma_{j}^{e}} G_{1}^{\prime}(x, y, s) d s-\psi_{j}^{e \prime} \int_{\Gamma_{j}^{e}} G_{1}(x, y, s) d s+\right. \\
\left.\quad+\eta_{j}^{e} \int_{\Gamma_{j}^{e}} G_{2}^{\prime}(x, y, s) d s-\eta_{j}^{e \prime} \int_{\Gamma_{j}^{e}} G_{2}(x, y, s) d s\right\},( \\
\chi(x, y) \eta^{e}(x, y)=\sum_{j=1}^{n}\left\{\eta_{j}^{e} \int_{\Gamma_{j}^{e}} G_{1}^{\prime}(x, y, s) d s-\eta_{j}^{e \prime} \int_{\Gamma_{j}^{e}} G_{1}(x, y, s) d s\right\} .
\end{aligned}
$$


Applying the expressions (40), (41) at the centers $\left(x_{c k}^{e}, y_{c k}^{e}\right)$ of the elements $\Gamma_{k}^{e}$ gives

$$
\begin{aligned}
& \sum_{j=1}^{n}\left\{\psi_{j}^{e} A_{k j}^{e}+\psi_{j}^{e \prime} B_{k j}^{e}+\eta_{j}^{e} C_{k j}^{e}+\eta_{j}^{e \prime} D_{k j}^{e}\right\}=0, \\
& \sum_{j=1}^{n}\left\{\eta_{j}^{e} A_{k j}^{e}+\eta_{j}^{e \prime} B_{k j}^{e}\right\}=0
\end{aligned}
$$

where

$$
\begin{array}{ll}
A_{k j}^{e}=\int_{\Gamma_{j}^{e}} G_{1}^{\prime}\left(x_{c k}^{e}, y_{c k}^{e}, s\right) d s-\beta_{k}^{e} \delta_{k j}, & B_{k j}^{e}=-\int_{\Gamma_{j}^{e}} G_{1}\left(x_{c k}^{e}, y_{c k}^{e}, s\right) d s, \\
C_{k j}^{e}=\int_{\Gamma_{j}^{e}} G_{2}^{\prime}\left(x_{c k}^{e}, y_{c k}^{e}, s\right) d s, & D_{k j}^{e}=-\int_{\Gamma_{j}^{e}} G_{2}\left(x_{c k}^{e}, y_{c k}^{e}, s\right) d s,
\end{array}
$$
$\beta_{k}^{e}=\beta^{e}\left(x_{c k}^{e}, y_{c k}^{e}\right)$ and $\delta_{k j}$ is the Kronecker delta.

To solve the boundary value problem (29)-(31) equation (29) is rewritten as two equations of second order

$$
\begin{gathered}
\Delta \psi^{i}=\eta^{i}, \\
\Delta \eta^{i}-S^{2} \eta^{i}=0 .
\end{gathered}
$$

where $\eta^{i}=-\omega^{i}$. The functions $\psi^{i}$ and $\eta^{i}$ satisfy the integral relations shown in equations (46), (47) see 34]

$$
\begin{aligned}
\chi(x, y) \psi^{i}(x, y)= & \int_{\Gamma^{i}}\left(\psi^{i}(s) G_{1}^{\prime}(x, y, s)-\psi^{i^{\prime}}(s) G_{1}(x, y, s)+\right. \\
& \left.+\eta^{i}(s) G_{4}^{\prime}(x, y, s)-\eta^{i^{\prime}}(s) G_{4}(x, y, s)\right) d s, \\
\chi(x, y) \eta^{i}(x, y)= & \int_{\Gamma^{i}}\left(\eta^{i}(s) G_{3}^{\prime}(x, y, s)-\eta^{i^{\prime}}(s) G_{3}(x, y, s)\right) d s .
\end{aligned}
$$

Where the Greens function $G_{1}$ is defined by (38), (39) and $G_{3}$ and $G_{4}$ are given by

$$
G_{3}=-K_{0}(S \rho), \quad G_{4}=\frac{1}{S^{2}}\left(G_{3}-G_{1}\right),
$$

where $K_{0}$ is the modified Bessel function of the second kind of zero order [35].

The boundary $\Gamma^{i}=\bigcup_{j=1}^{m} \Gamma_{j}^{i}$ is approximated as the sum of linear segments ${ }_{205} \Gamma_{j}^{i}$. The functions $\psi^{i}(s), \psi^{i^{\prime}}(s), \eta^{i}(s), \eta^{i^{\prime}}(s)$ are approximated by the piecewise 
constant functions with the values $\psi_{j}^{i}, \psi_{j}^{i^{\prime}}, \eta_{j}^{i}, \eta_{j}^{i^{\prime}}$ on the segments $\Gamma_{j}^{i}$ respectively. Then the equations (46), (47) are rewritten in the form

$$
\begin{aligned}
\chi(x, y) \psi^{i}(x, y)=\sum_{j=1}^{m}\left\{\psi_{j}^{i} \int_{\Gamma_{j}^{i}} G_{1}^{\prime}(x, y, s) d s-\psi_{j}^{i^{\prime}} \int_{\Gamma_{j}^{i}} G_{1}(x, y, s) d s+\right. \\
\left.+\eta_{j}^{i} \int_{\Gamma_{j}^{i}} G_{4}^{\prime}(x, y, s) d s-\eta_{j}^{i^{\prime}} \int_{\Gamma_{j}^{i}} G_{4}(x, y, s) d s\right\}, \\
\chi(x, y) \eta^{i}(x, y)=\sum_{j=1}^{m}\left\{\eta_{j}^{i} \int_{\Gamma_{j}^{i}} G_{3}^{\prime}(x, y, s) d s-\eta_{j}^{i^{\prime}} \int_{\Gamma_{j}^{i}} G_{3}(x, y, s) d s\right\} .
\end{aligned}
$$

Substituting the centers $\left(x_{c k}^{i}, y_{c k}^{i}\right)$ of the segments $\Gamma_{k}^{i}$ into the relations (49), (50) gives

$$
\begin{aligned}
& \sum_{j=1}^{m}\left\{\psi_{j}^{i} A_{k j}^{i}+\psi_{j}^{i^{\prime}} B_{k j}^{i}+\eta_{j}^{i} C_{k j}^{i}+\eta_{j}^{i^{\prime}} D_{k j}^{i}\right\}=0, \\
& \sum_{j=1}^{m}\left\{\eta_{j}^{i} E_{k j}^{i}+\eta_{j}^{i^{\prime}} F_{k j}^{i}\right\}=0
\end{aligned}
$$

where

$$
\begin{array}{ll}
A_{k j}^{i}=\int_{\Gamma_{j}^{i}} G_{1}^{\prime}\left(x_{c k}^{i}, y_{c k}^{i}, s\right) d s-\beta_{k}^{i} \delta_{k j}, & B_{k j}^{i}=-\int_{\Gamma_{j}^{i}} G_{1}\left(x_{c k}^{i}, y_{c k}^{i}, s\right) d s, \\
C_{k j}^{i}=\int_{\Gamma_{j}^{i}} G_{4}^{\prime}\left(x_{c k}^{i}, y_{c k}^{i}, s\right) d s, & D_{k j}^{i}=-\int_{\Gamma_{j}^{i}} G_{4}\left(x_{c k}^{i}, y_{c k}^{i}, s\right) d s, \\
E_{k j}^{i}=\int_{\Gamma_{j}^{i}} G_{3}^{\prime}\left(x_{c k}^{i}, y_{c k}^{i}, s\right) d s-\beta_{k}^{i} \delta_{k j}, & F_{k j}^{i}=-\int_{\Gamma_{j}^{i}} G_{3}\left(x_{c k}^{i}, y_{c k}^{i}, s\right) d s,
\end{array}
$$$$
\beta_{k}^{i}=\beta\left(x_{c k}^{i}, y_{c k}^{i}\right) .
$$

To close the obtained system (42), (51) of linear algebraic equations (SLAE) the relations from the boundary conditions are used. The conditions (25), (26) are written in the form

$$
\begin{array}{lll}
\psi_{j}^{e}=y_{c j}^{e}, & \eta_{j}=0 & \text { on } A D \\
\psi_{j}^{e}=\psi_{k}^{e}, & \psi_{j}^{e \prime}=-\psi_{k}^{e \prime}, \quad \eta_{j}^{e}=\eta_{k}^{e}, \quad \eta_{j}^{e \prime}=-\eta_{k}^{e} & \text { on } A E \text { and } D F
\end{array}
$$

where $j$ and $k$ are the indexes of the corresponded linear segments on the sides 
$A E$ and $D F$. The symmetry conditions (27), (28), (30) are presented as

$$
\begin{aligned}
& \psi_{j}^{e}=h_{1}, \quad \eta_{j}^{e}=0 \quad \text { on } E F, \\
& \psi_{j}^{e}=0, \quad \eta_{j}^{e}=0 \quad \text { on } A B \text { and } C D \text {, } \\
& \psi_{j}^{i}=0, \quad \eta_{j}^{i}=0 \quad \text { on BOC. }
\end{aligned}
$$

The conditions (31) on the boundary $B C$ of the porous medium and free space interface are written in the form

$$
\psi_{j}^{e}=\psi_{k}^{i}, \quad \psi_{j}^{e^{\prime}}=-\psi_{k}^{i^{\prime}}, \quad \eta_{j}^{e \prime}=S^{2} \psi_{k}^{i^{\prime}}-\eta_{k}^{i^{\prime}}, \quad \eta_{j}^{e}=\eta_{k}^{i},
$$

where $j$ and $k$ are the indexes of the matching linear segments $\Gamma_{j}^{e}$ and $\Gamma_{k}^{i}$ on $B C$.

The SLAE obtained is then solved numerically to find the unknown quantities $\psi_{j}^{e}, \psi_{j}^{e^{\prime}}, \eta_{j}^{e}, \eta_{j}^{e^{\prime}}, j=\overline{1, n} ; \psi_{j}^{i}, \psi_{j}^{i^{\prime}}, \eta_{j}^{i}, \eta_{j}^{i^{\prime}}, j=\overline{1, m}$. The functions $\psi^{e}(x, y), \omega^{e}(x, y), \psi^{i}(x, y), \omega^{i}(x, y)$ at the general point $(x, y)$ can then be calculated from the formulas (40), (41), (49), (50). The formulas for the velocity components are written as

$$
\begin{aligned}
\chi(x, y) v_{x}^{e}(x, y)= & \sum_{j=1}^{n}\left\{\psi_{j}^{e} \int_{\Gamma_{j}^{e}} \frac{\partial G_{1}^{\prime}(x, y, s)}{\partial y} d s-\psi_{j}^{e \prime} \int_{\Gamma_{j}^{e}} \frac{\partial G_{1}(x, y, s)}{\partial y} d s+\right. \\
& \left.+\eta_{j}^{e} \int_{\Gamma_{j}^{e}} \frac{\partial G_{2}^{\prime}(x, y, s)}{\partial y} d s-\eta_{j}^{e \prime} \int_{\Gamma_{j}^{e}} \frac{\partial G_{2}(x, y, s)}{\partial y} d s\right\}, \\
\chi(x, y) v_{y}^{e}(x, y)= & -\sum_{j=1}^{n}\left\{\psi_{j}^{e} \int_{\Gamma_{j}^{e}} \frac{\partial G_{1}^{\prime}(x, y, s)}{\partial x} d s-\psi_{j}^{e^{\prime}} \int_{\Gamma_{j}^{e}} \frac{\partial G_{1}(x, y, s)}{\partial x} d s+\right. \\
& \left.+\eta_{j}^{e} \int_{\Gamma_{j}^{e}} \frac{\partial G_{2}^{\prime}(x, y, s)}{\partial x} d s-\eta_{j}^{e \prime} \int_{\Gamma_{j}^{e}} \frac{\partial G_{2}(x, y, s)}{\partial x} d s\right\} \\
\chi(x, y) v_{x}^{i}(x, y)= & \sum_{j=1}^{m}\left\{\psi_{j}^{i} \int_{\Gamma_{j}^{i}} \frac{\partial G_{1}^{\prime}(x, y, s)}{\partial y} d s-\psi_{j}^{i^{\prime}} \int_{\Gamma_{j}^{i}} \frac{\partial G_{1}(x, y, s)}{\partial y} d s+\right. \\
& \left.+\eta_{j}^{i} \int_{\Gamma_{j}^{i}} \frac{\partial G_{4}^{\prime}(x, y, s)}{\partial y} d s-\eta_{j}^{i^{\prime}} \int_{\Gamma_{j}^{i}} \frac{\partial G_{4}(x, y, s)}{\partial y} d s\right\}
\end{aligned}
$$




$$
\begin{aligned}
\chi(x, y) v_{y}^{i}(x, y)= & -\sum_{j=1}^{m}\left\{\psi_{j}^{i} \int_{\Gamma_{j}^{i}} \frac{\partial G_{1}^{\prime}(x, y, s)}{\partial x} d s-\psi_{j}^{i^{\prime}} \int_{\Gamma_{j}^{i}} \frac{\partial G_{1}(x, y, s)}{\partial x} d s+\right. \\
& \left.+\eta_{j}^{i} \int_{\Gamma_{j}^{i}} \frac{\partial G_{4}^{\prime}(x, y, s)}{\partial x} d s-\eta_{j}^{i^{\prime}} \int_{\Gamma_{j}^{i}} \frac{\partial G_{4}(x, y, s)}{\partial x} d s\right\} .
\end{aligned}
$$

The main numerical difficulties of the BEM described above are connected with the calculation of the integrals

$$
\begin{aligned}
& \int G_{k} d s, \quad \int G_{k}^{\prime} d s, \quad \int \frac{\partial G_{k}}{d x} d s, \\
& \int \frac{\partial G_{k}^{\prime}}{d y} d s, \quad \int \frac{\partial G_{k}}{d y} d s, \quad \int \frac{\partial G_{k}^{\prime}}{d y} d s
\end{aligned}
$$

on the linear segments $\Gamma_{j}^{e}$ and $\Gamma_{j}^{i}$, where $k=\overline{1,4}$. The analytical formulas for the integrals (60) for $k=1,2$ are given in [31]. For $k=3$ the integrals are determined numerically and for $k=4$ the integrals are found by combining the integrals for $k=1,3$ using (48). To calculate the integrals involving the derivative of Greens function for $k=3$ the following formulas with equation (39) are used:

$$
\begin{aligned}
\frac{d G_{3}}{d x}=- & \frac{S K_{1}(S \rho)\left(x_{1}-x\right)}{\rho}, \quad \frac{d G_{3}}{d y}=-\frac{S K_{1}(S \rho)\left(y_{1}-y\right)}{\rho} \\
\frac{d G_{3}^{\prime}}{d x}= & \frac{S}{\rho}\left[\left(S K_{0}(S \rho)+\frac{2 K_{1}(S \rho)}{\rho}\right) \frac{\left(x_{1}-x\right)}{\rho} \times\right. \\
& \left.\quad \times\left(\left(x_{1}-x\right) \sin \theta-\left(y_{1}-y\right) \cos \theta\right)-K_{1}(S \rho) \sin \theta\right]
\end{aligned}
$$

235

$$
\begin{aligned}
\frac{d G_{3}^{\prime}}{d y}=\frac{S}{\rho}[( & \left.S K_{0}(S \rho)+\frac{2 K_{1}(S \rho)}{\rho}\right) \frac{\left(y_{1}-y\right)}{\rho} \times \\
& \left.\times\left(\left(x_{1}-x\right) \sin \theta-\left(y_{1}-y\right) \cos \theta\right)+K_{1}(S \rho) \cos \theta\right],
\end{aligned}
$$

where $\theta$ is the angle of the tangent to the current integration linear segment and $K_{1}$ is the modified Bessel function of the second kind of first order.

\section{Analytical solution}

For the circular porous cylinder in a circular cell with Kuwabara conditions

the analytical solution was obtained in [13]. For the free space flow the stream 
function can be written in the form

$$
\psi_{a}^{e}(r, \theta)=\left(\frac{A_{1}}{r}+B_{1} r+C_{1} r \ln r+D_{1} r^{3}\right) \sin \theta,
$$

For the porous domain the stream function can be written as

$$
\psi_{a}^{i}(r, \theta)=\left(B_{2} r+C_{2} I_{1}(S r)\right) \sin \theta,
$$

where $I_{1}$ is the modified Bessel function of the first kind of first order. The functions (64) and (65) take into account the boundary conditions (28), (30).

The unknown coefficients in (64) and (65) are found from the SLAE obtained from the boundary conditions (25), (31). The resulting relationships are shown below:

$$
\begin{aligned}
\alpha A_{1}+B_{1}-\frac{\ln \alpha}{2} C_{1}+\frac{1}{\alpha} D_{1} & =1, \\
\alpha C_{1}+4 D_{1} & =0, \\
A_{1}+B_{1}+D_{1}-B_{2}-I_{1}(S) C_{2} & =0, \\
-A_{1}+B_{1}+C_{1}+3 D_{1}-B_{2}+\left(I_{1}(S)-S I_{0}(S)\right) C_{2} & =0, \\
2 C_{1}-8 D_{1}-S^{2} B_{2} & =0, \\
2 C_{1}+8 D_{1}-S^{2} I_{1}(S) C_{2} & =0,
\end{aligned}
$$

where $I_{0}$ is the modified Bessel function of the first kind of zero order and the solidity $\alpha$ is given by $\alpha=h^{-2}$. The velocity components and vorticity in polar coordinates are found from the following formulae

$$
\begin{aligned}
v_{r a}^{e}(r, \theta) & =\frac{1}{r} \frac{\partial \psi_{a}^{e}}{\partial \theta}=\left(\frac{A_{1}}{r^{2}}+B_{1}+C_{1} \ln r+D_{1} r^{2}\right) \cos \theta \\
v_{r a}^{i}(r, \theta) & =\frac{1}{r} \frac{\partial \psi_{a}^{i}}{\partial \theta}=\left(B_{2}+C_{2} \frac{I_{1}(S r)}{r}\right) \cos \theta \\
v_{\theta a}^{e}(r, \theta) & =-\frac{\partial \psi_{a}^{e}}{\partial r}=-\left(-\frac{A_{1}}{r^{2}}+B_{1}+C_{1}(\ln r+1)+3 D_{1} r^{2}\right) \sin \theta \\
v_{\theta a}^{i}(r, \theta) & =-\frac{\partial \psi_{a}^{i}}{\partial r}=-\left(B_{2}+C_{2}\left(I_{0}(S)-\frac{I_{1}(S)}{S r}\right)\right) \sin \theta \\
\omega_{a}^{e}(r, \theta) & =-\left(\frac{1}{r} \frac{\partial}{\partial r}\left(r \frac{\partial \psi_{a}^{e}}{\partial r}\right)+\frac{1}{r^{2}} \frac{\partial^{2} \psi_{a}^{e}}{\partial \theta^{2}}\right)=-2\left(\frac{C_{1}}{r}+4 D_{1} r\right) \sin \theta \\
\omega_{a}^{i}(r, \theta) & =-\left(\frac{1}{r} \frac{\partial}{\partial r}\left(r \frac{\partial \psi_{a}^{i}}{\partial r}\right)+\frac{1}{r^{2}} \frac{\partial^{2} \psi_{a}^{i}}{\partial \theta^{2}}\right)=-S^{2} C_{2} I_{1}(S r) \sin \theta
\end{aligned}
$$




\section{Numerical results}

The fluid flow through a porous circular cylinder in a circular Kuwabara cell with porosity $\varepsilon=0.96(h=5)$ was studied to test the model developed. The number of linear elements on the boundaries $\Gamma^{e}$ and $\Gamma^{i}$ is taken to be $n=161$ and $m=50$ respectively. The integration step for calculating the integrals (60) at $k=3,4$ is taking to be $10^{-4}$.

To estimate the accuracy of the developed method the absolute $E_{\psi}(x, y)$, $E_{v x}(x, y), E_{v y}(x, y), E_{\omega}(x, y)$ and relative $\varepsilon_{\psi}, \varepsilon_{v x}, \varepsilon_{v y}, \varepsilon_{\omega}$ errors were calculated for the functions $\psi(x, y), v_{x}(x, y), v_{y}(x, y), \omega(x, y)$ respectively, where:

$$
\begin{array}{r}
E_{\psi}(x, y)=\left|\psi(x, y)-\psi_{a}(x, y)\right|, \quad E_{v x}(x, y)=\left|v_{x}(x, y)-v_{x a}(x, y)\right|, \\
E_{v y}(x, y)=\left|v_{y}(x, y)-v_{y a}(x, y)\right|, \quad E_{\omega}(x, y)=\left|\omega(x, y)-\omega_{a}(x, y)\right|, \\
\varepsilon_{\psi}=\frac{\max E_{\psi}(x, y)}{\max \left|\psi_{a}(x, y)\right|}, \quad \varepsilon_{v x}=\frac{\max E_{v x}(x, y)}{\max \sqrt{v_{x a}^{2}(x, y)+v_{y a}^{2}(x, y)}}, \\
\varepsilon_{v y}=\frac{\max E_{v y}(x, y)}{\max \sqrt{v_{x a}^{2}(x, y)+v_{y a}^{2}(x, y)}}, \quad \varepsilon_{\omega}=\frac{\max E_{\omega}(x, y)}{\max \left|\omega_{a}(x, y)\right|} .
\end{array}
$$

The streamlines and vorticity distributions at $S=3$ and 6 are shown in Fig. 3, It is seen from the figure that the flow rate through the porous cylinder decreases with the growth of $S$ (decreasing the permeability of the porous medium). For larger $S$ the vorticity near the cylinder boundary is larger. The distributions of $E_{\psi}(x, y), E_{v x}(x, y), E_{v y}(x, y), E_{\omega}(x, y)$ for $S=3$ are shown in Fig. 4. The values of the relative errors $\varepsilon_{\psi}, \varepsilon_{v x}, \varepsilon_{v y}, \varepsilon_{\omega}$ for $S=1,3,6,10$ are given in Table 1. It is seen from the figure and table that the method developed provides good accuracy with a relatively small number of linear elements on the flow boundary. It can also be observed that smaller values of absolute and relative errors are obtained for the stream function. The corresponding values for the velocity components and vorticity are higher due to additional numerical differentiation. Larger values of the errors are observed in the center of the domain $\Omega^{e}$ for the stream function and at the interface between the free space and the porous medium for for the velocity components and vorticity (Fig. (4). 

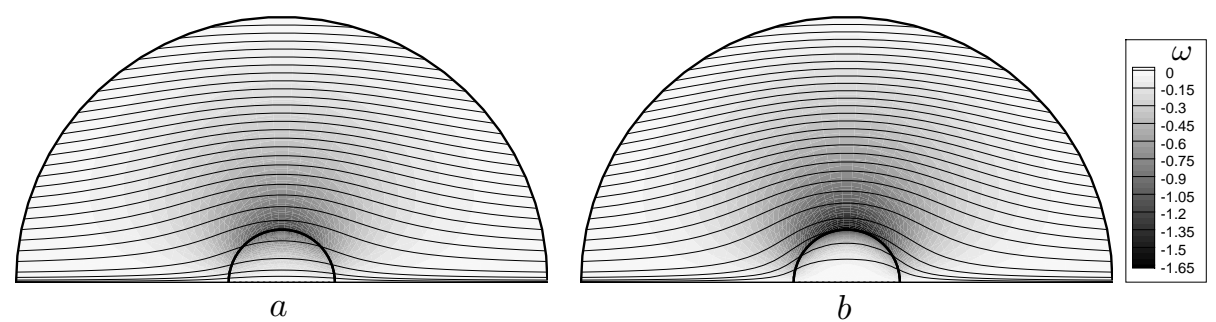

Figure 3: Streamlines and vorticity distributions in circular cell for $S=3(a), S=6(b)$
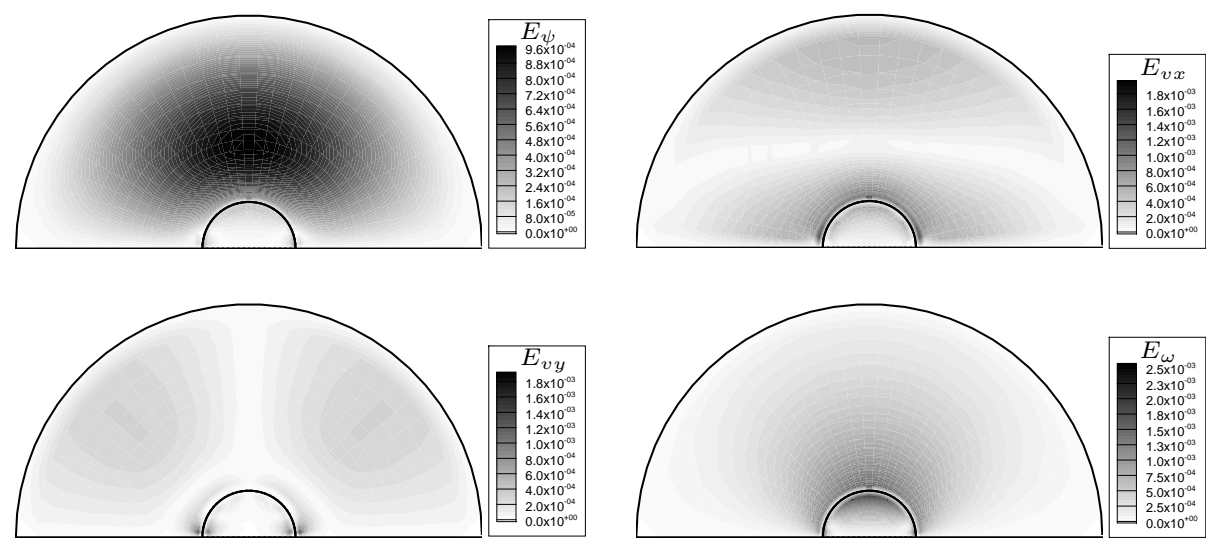

Figure 4: The distributions $E_{\psi}(x, y), E_{v x}(x, y), E_{v y}(x, y), E_{\omega}(x, y)$ in circular cell for $S=3$

Table 1: Relative errors $\varepsilon_{\psi}, \varepsilon_{v x}, \varepsilon_{v y}, \varepsilon_{\omega}$

\begin{tabular}{|c|c|c|c|c|}
\hline$S$ & $\varepsilon_{\psi} \times 10^{4}$ & $\varepsilon_{v x} \times 10^{3}$ & $\varepsilon_{v y} \times 10^{3}$ & $\varepsilon_{\omega} \times 10^{3}$ \\
\hline 1 & 3.519 & 3.304 & 5.216 & 2.317 \\
3 & 1.951 & 1.464 & 1.461 & 2.039 \\
6 & 2.173 & 1.043 & 0.370 & 3.183 \\
10 & 2.177 & 1.085 & 0.367 & 3.470 \\
\hline
\end{tabular}




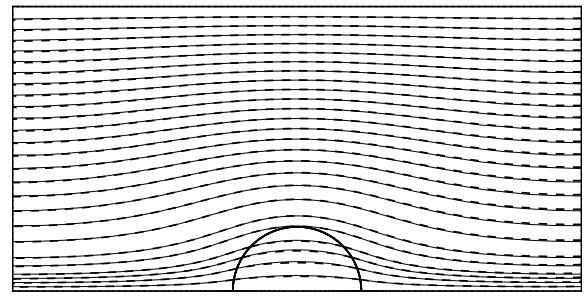

$a$

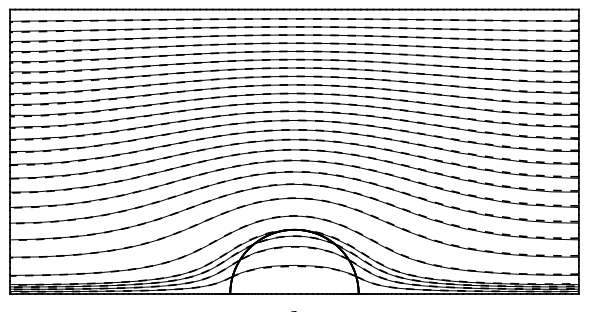

$b$

Figure 5: Fluid flow streamlines in rectangular cell with porosity $\varepsilon=0.96$ for $S=3(a)$ and $S=6(b)$. The solid and dotted lines correspond to the BEM and FVM models respectively.

The mathematical model was also applied to the fluid flow through a porous cylinder in a rectangular cell. Such a model is a more accurate fluid flow description but it is not possible to obtain an analytical solution for comparison. The periodic boundary conditions (26) on the outer left and right sides of the cell and the symmetry conditions (27), (28) on the top and bottom sides are applied. The results obtained are compared with the results obtained by ANSYS/FLUENT where the boundary value problem was solved for the NavierStokes-Brinkman approach using the FVM [36]. The fluid flow streamlines for the rectangular cell with porosity $\varepsilon=0.96$ for two values of $S$ are given in Fig. 5 , For this porosity as defined by (23) the cell dimensions are $h_{1}=h_{2}=4.43$ $\left(\alpha=\pi / 4 h_{1} h_{2}\right)$. The density of the streamlines is higher near the symmetry axis. As can be seen in the figures there is no significant difference between the streamlines from the two approaches.

The coefficient of fluid capture $Q=\psi(0,1)$ as a function of $S$ for various values of $\varepsilon$ has been calculated. In Fig. 6, $a$ and in Table 2 the dependencies $Q(S)$ found by the BEM and the FVM are shown. Good agreement between the two models is observed. A comparison of the dependencies $Q(S)$ obtained numerically by the BEM in a rectangular cell and from the analytical solution (64), (65) for a circular cell is shown in Fig. 6, $b$ and in Table 2, In this case the dependencies $Q(S)$ for the rectangular and circular periodic cells agree well for larger values of the porosity $\varepsilon=0.9,0.96,0.99$. The values presented in Table 2 

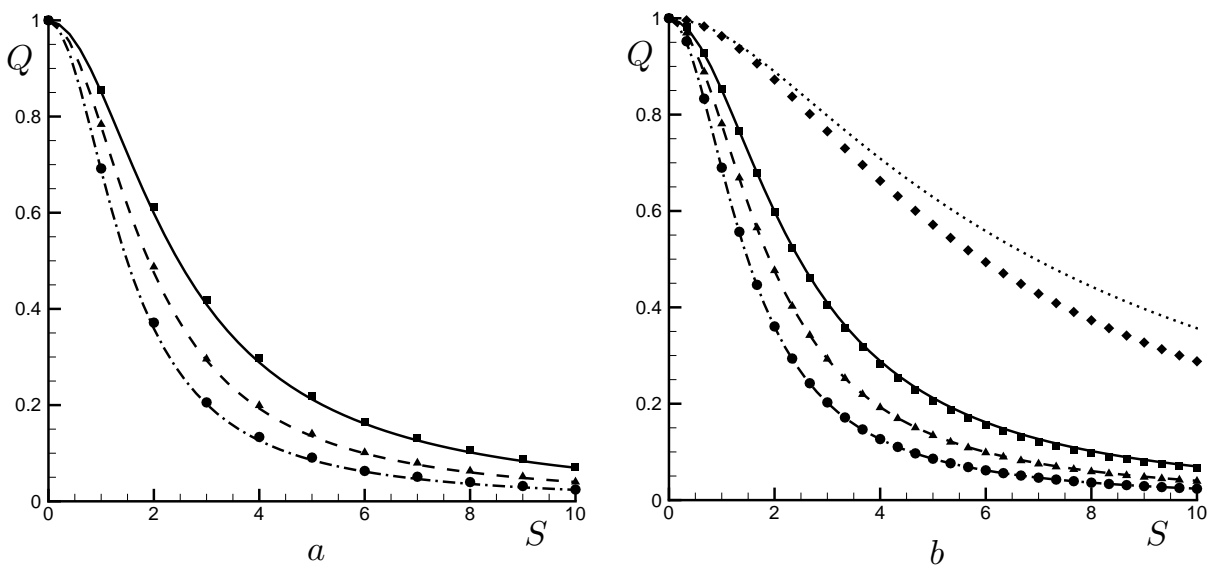

Figure 6: The coefficient of fluid capture $Q(S)$ for the rectangular cell calculated using BEM (solid lines $-\varepsilon=0.9$, dashed lines $-\varepsilon=0.96$, dot-dashed lines $-\varepsilon=0.99$ ). The symbols correspond to FVM calculation $(a)$ and analytical solution for circular cell (b): square symbols $-\varepsilon=0.9$, triangles $-\varepsilon=0.96$, circles $-\varepsilon=0.99)$. The dotted line and rhombus symbols $(b)$ corresponds to $\varepsilon=0.6$ for numerical solution in rectangular and analytical solution in circular cells respectively.

Table 2: The coefficient $Q(S)$ for circular cylinder in circular and rectangular cells

\begin{tabular}{|c|c|c|c|c|c|c|c|c|c|}
\hline \multirow{3}{*}{$S$} & \multicolumn{3}{|c|}{$\varepsilon=0.9$} & \multicolumn{3}{c|}{$\varepsilon=0.96$} & \multicolumn{3}{c|}{$\varepsilon=0.99$} \\
\cline { 2 - 10 } & $\begin{array}{c}\text { formulas } \\
\text { (64), (65) }\end{array}$ & FVM & BEM & $\begin{array}{c}\text { formulas } \\
\text { (64), (65) }\end{array}$ & FVM & BEM & $\begin{array}{c}\text { formulas } \\
\text { (64), (65) }\end{array}$ & FVM & BEM \\
\hline 1 & 0.8523 & 0.8543 & 0.8515 & 0.7812 & 0.7839 & 0.7794 & 0.6899 & 0.692 & 0.6880 \\
3 & 0.4053 & 0.4190 & 0.4092 & 0.2928 & 0.2961 & 0.2930 & 0.2027 & 0.2056 & 0.2021 \\
6 & 0.1559 & 0.1647 & 0.1615 & 0.0986 & 0.1015 & 0.0997 & 0.0618 & 0.0631 & 0.0617 \\
10 & 0.0658 & 0.0708 & 0.0698 & 0.0393 & 0.0404 & 0.0402 & 0.0237 & 0.0245 & 0.0238 \\
\hline
\end{tabular}



problems of fluid flow through the porous square and triangular cylinders in a rectangular periodic cell have been considered. The cell dimensions are $h_{1}=$ $h_{2}=4.43$. The square and triangular domains can be defined as $\Omega_{1}:\{-1<$ $x<1,0<y<1\}, \Omega_{2}:\{-1<x<1, y>0, y<0.5(x+1)\}$ respectively. applied. The fluid flow streamlines for $S=10,200$ from the cell model are given in Fig. 8. It is seen that BEM and FVM approaches give very similar results. 


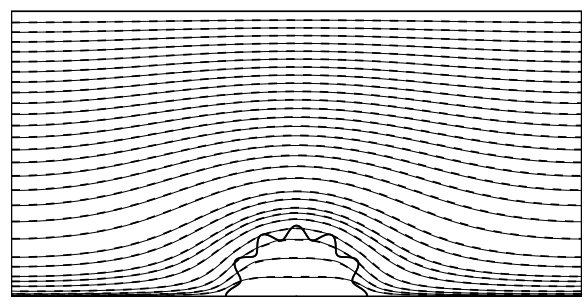

$a$

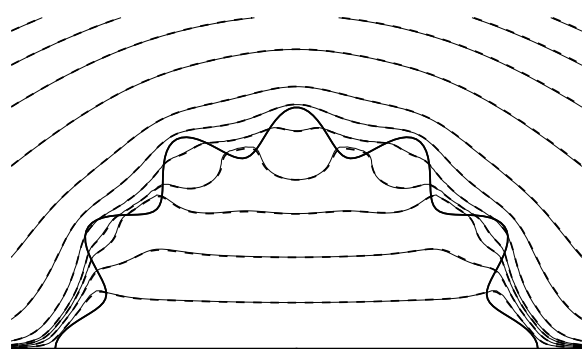

$c$
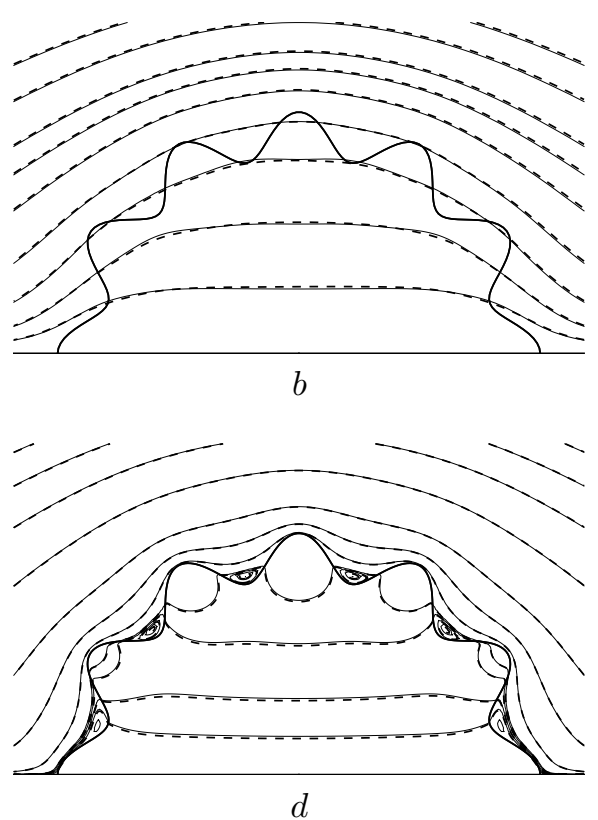

Figure 7: Fluid flow through "idealized capsid" streamlines for the rectangular cell with $h_{1}=h_{2}=4.43$ at $S=10(a, b), 50(c), 200(d)$. The solid and dotted lines correspond to the BEM and FVM models respectively.

The absolute $E_{\psi}^{n}(x, y)$ and relative $\varepsilon_{\psi}^{n}$ differences between the functions $\psi_{1}(x, y)$ and $\psi_{2}(x, y)$ obtained from the BEM and FVM solutions respectively were calculated from the formulae

$$
E_{\psi}^{n}(x, y)=\left|\psi_{1}(x, y)-\psi_{2}(x, y)\right|, \quad \varepsilon_{\psi}^{n}=\frac{\max E_{\psi}^{n}(x, y)}{\max \left|\psi_{2}(x, y)\right|} .
$$

The calculated values of the relative differences between the BEM and FVM solutions $\varepsilon_{\psi}^{n}$ for $S=10,200$ are presented in Table 3. It is seen that both methods give very similar results.

The coefficient of fluid capture $Q=\psi\left(x^{*}, y^{*}\right)\left(x^{*}=0, y^{*}=1.1-\right.$ capsid model, $x^{*}=-1, y^{*}=1-$ square cylinder, $x^{*}=1, y^{*}=1-$ triangular cylinder) for $S=10,200$ has been calculated. The corresponding values of $Q$ for the fluid flow are given in Table4 It is seen from the table that good correlation between the BEM and FVM is obtained for all three bodies. 

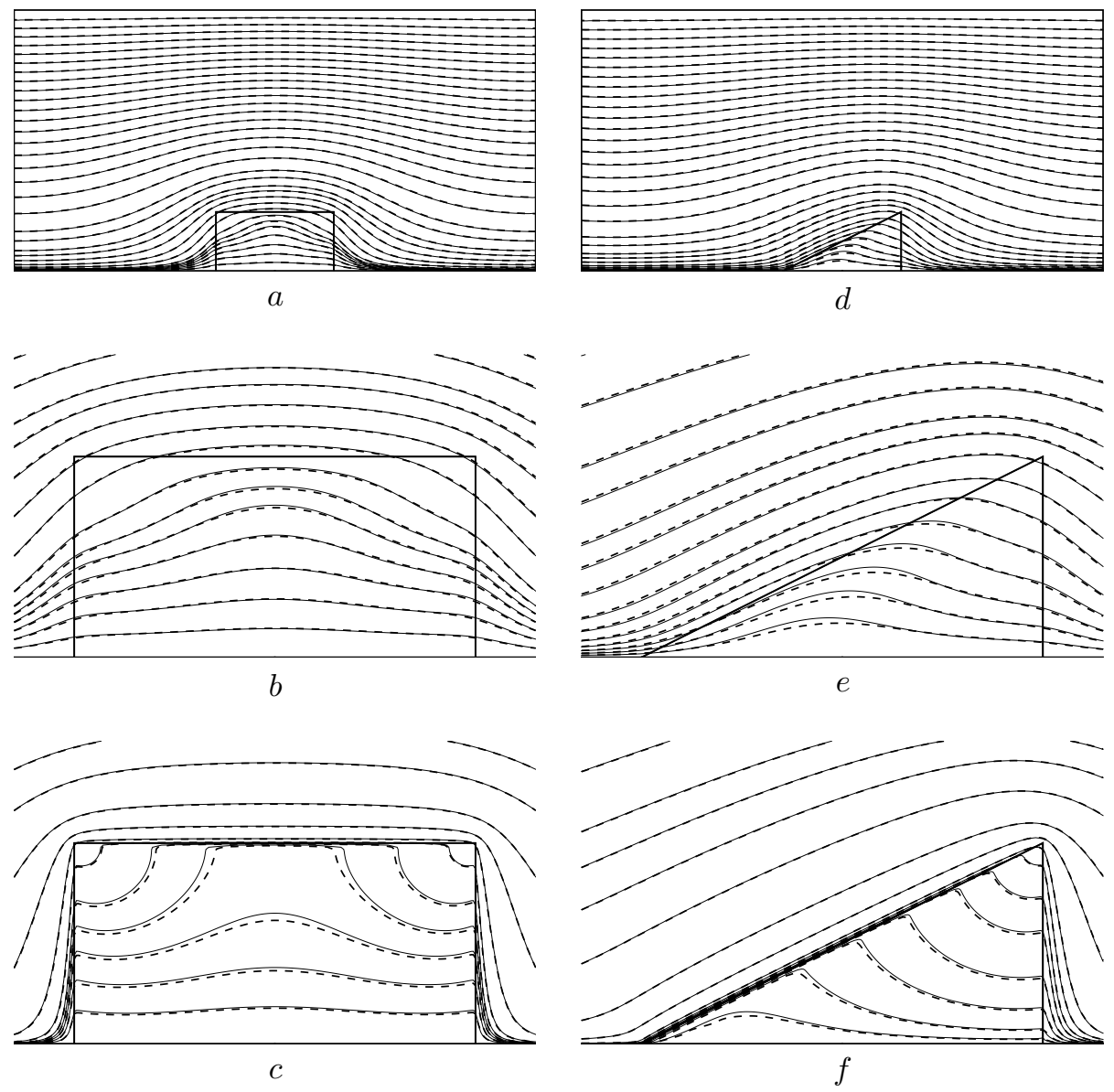

Figure 8: Fluid flow through square $(\mathrm{a}, \mathrm{b}, \mathrm{c})$ and triangular $(\mathrm{d}, \mathrm{e}, \mathrm{f})$ cylinders streamlines for the rectangular cell with $h_{1}=h_{2}=4.43$ at $S=10$ (a,b,d,e) and $S=200$ (c,f). The solid and dotted lines correspond to the BEM and FVM models respectively.

Table 3: Relative difference $\varepsilon_{\psi}^{n}$ between BEM and FVM solutions

\begin{tabular}{|c|c|c|c|}
\hline$S$ & capsid & square cylinder & triangular cylinder \\
\hline 10 & 0.002448 & 0.002973 & 0.002075 \\
200 & 0.001692 & 0.003262 & 0.002014 \\
\hline
\end{tabular}


Table 4: The coefficient of fluid capture $Q(S)$ for capsid, square and triangular cylinders in rectangular cell

\begin{tabular}{|c|c|c|c|c|c|c|}
\hline \multirow{2}{*}{$S$} & \multicolumn{2}{|c|}{ capsid } & \multicolumn{2}{c|}{ square cylinder } & \multicolumn{2}{c|}{ triangular cylinder } \\
\cline { 2 - 7 } & FVM & BEM & FVM & BEM & FVM & BEM \\
\hline 10 & 0.0611 & 0.06133 & 0.06728 & 0.06885 & 0.1175 & 0.1139 \\
200 & 0.00036 & 0.0003229 & 0.0007547 & 0.0007420 & 0.001499 & 0.001445 \\
\hline
\end{tabular}

\section{Acknowledgement}

The work was performed in the frameworks of the Russian Government Program of Competitive Growth at Kazan Federal University and supported by the Russian Foundation for Basic Research (grants No. 15-01-06135, 16-51- 


\section{References}

[1] V. Kirsh, Stokes flow past periodic rows of porous cylinders, Theoretical Foundations of Chemical Engineering 40 (5) (2006) 465-471. doi:10.1134/S0040579506050034.

URL http://dx .doi .org/10 .1134/S0040579506050034

[2] S. Zaripov, O. Soloveva, S. Solovev, Inertial deposition of aerosol particles in a periodic row of porous cylinders, Aerosol Science and Technology 49 (2015) 400-408. doi:10.1080/02786826.2015.1036834.

URL http://www .tandfonline.com/doi/full/10.1080/02786826.2015.1036834

[3] S. Dunnett, C. Clement, A numerical model of fibrous filters containing deposit, Engineering Analysis with Boundary Elements 33 (5) (2009) 601610. doi:http://dx.doi.org/10.1016/j.enganabound.2008.10.010. URL http://www.sciencedirect.com/science/article/pii/S0955799708001963

[4] L. von Wolfersdorf, W. Monch, Potential flow past a porous circular cylinder, ZAMM - Journal of Applied Mathematics and Mechanics / Zeitschrift fur Angewandte Mathematik und Mechanik $80 \quad$ (7) (2000) 457-471. doi:10.1002/1521-4001(200007)80:7<457: :AID-ZAMM457>3.0.CO;2-U. URL http://dx.doi.org/10.1002/1521-4001(200007)80:7<457: :AID-ZAMM457>3.0.C0;2-U

[5] G. S. Beavers, D. D. Joseph, Boundary conditions at a naturally permeable wall, Journal of Fluid Mechanics 30 (1967) 197-207. doi:10.1017/S0022112067001375. URL http://journals . cambridge.org/article_S0022112067001375

[6] P. G. Saffman, On the boundary condition at the surface of a porous medium, Studies in Applied Mathematics 50 (2) (1971) 93-101. doi:10.1002/sapm197150293.

URL http://dx.doi.org/10.1002/sapm197150293 
[7] G. Neale, W. Nader, Practical significance of Brinkman's extension of Darcy's law: Coupled parallel flows within a channel and a bounding porous medium, The Canadian Journal of Chemical Engineering 52 (4) (1974) 475478. doi:10.1002/cjce.5450520407

URL http://dx.doi.org/10.1002/cjce.5450520407

[8] S. Haber, R. Mauri, Boundary conditions for Darcy's flow through porous media, International Journal of Multiphase Flow 9 (5) (1983) 561-574. doi:http://dx.doi.org/10.1016/0301-9322(83)90018-6 URL http://www.sciencedirect.com/science/article/pii/0301932283900186

[9] K. Vafai, R. Thiyagaraja, Analysis of flow and heat transfer at the interface region of a porous medium, International Journal of Heat and Mass Transfer 30 (7) (1987) 1391-1405. doi:http://dx.doi.org/10.1016/0017-9310(87)90171-2

URL http://www.sciencedirect.com/science/article/pii/0017931087901712

[10] M. Sahraoui, M. Kaviany, Slip and no-slip velocity boundary conditions at interface of porous, plain media, International Journal of Heat and Mass Transfer 35 (4) (1992) 927-943. doi:http://dx.doi.org/10.1016/0017-9310(92)90258-T URL http://www.sciencedirect.com/science/article/pii/001793109290258T

[11] J. Ochoa-Tapia, S. Whitaker, Momentum transfer at the boundary between a porous medium and a homogeneous fluidi. theoretical development, International Journal of Heat and Mass Transfer 38 (14) (1995) 2635-2646. doi:http://dx.doi.org/10.1016/0017-9310(94)00346-W URL http://www.sciencedirect.com/science/article/pii/001793109400346W

[12] J. Ochoa-Tapia, S. Whitaker, Momentum transfer at the boundary between a porous medium and a homogeneous fluidii. comparison with experiment, International Journal of Heat and Mass Transfer 38 (14) (1995) 2647-2655. doi:http://dx.doi.org/10.1016/0017-9310(94)00347-X. URL http://www.sciencedirect.com/science/article/pii/001793109400347X 
[13] I. B. Stechkina, Drag of porous cylinders in a viscous fluid at low Reynolds numbers, Fluid Dynamics 14 (6) (1979) 912-915. doi:10.1007/BF01051997.

URL http://dx.doi .org/10.1007/BF01051997

[14] S. Kuwabara, The forces experienced by randomly distributed parallel circular cylinders or spheres in a viscous flow at small Reynolds numbers, Journal of Physical Society of Japan 14 (4) (1959) 527-532.

[15] J. Happel, H. Brenner, Low Reynolds Number Hydrodynamics: with special applications to particulate media, Prentice-Hall, Englewood Cliffs, New York, 1965.

[16] H. C. Brinkman, A calculation of the viscous force exerted by a flowing fluid on a dense swarm of particles, Appl.Sci.Res. A1 (1) (1947) 27-34.

[17] S. Deo, P. K. Yadav, A. Tiwari, Slow viscous flow through a membrane built up from porous cylindrical particles with an impermeable core, Applied Mathematical Modelling 34 (5) (2010) 1329-1343. doi:http://dx.doi.org/10.1016/j.apm.2009.08.014. URL http://www.sciencedirect.com/science/article/pii/S0307904X09002601

[18] S. Deo, A. Filippov, A. Tiwari, S. Vasin, V. Starov, Hydrodynamic perneability of aggregates of porous particles with an impermeable core, Advances in Colloid and Interface Science 164 (1-2) (2011) 21-37. doi:http://dx.doi.org/10.1016/j.cis.2010.08.004. URL http://www . sciencedirect.com/science/article/pii/S0001868610001466

[19] S. I. Vasin, A. N. Filippov, Cell models for flows in concentrated media composed of rigid impenetrable cylinders covered with a porous layer, Colloid Journal 71 (2) (2009) 141-155. doi:10.1134/S1061933X0902001X. URL http://dx.doi .org/10.1134/S1061933X0902001X

[20] J. H. Masliyah, M. Polikar, Terminal velocity of porous spheres, The Canadian Journal of Chemical Engineering 58 (3) (1980) 299-302. 
doi:10.1002/cjce.5450580303.

URL http://dx.doi.org/10.1002/cjce.5450580303

[21] K. Nandakumar, J. H. Masliyah, Laminar flow past a permeable sphere, The Canadian Journal of Chemical Engineering 60 (2) (1982) 202-211. doi:10.1002/cjce.5450600202.

URL http://dx.doi.org/10.1002/cjce.5450600202

[22] P. D. Noymer, L. R. Glicksman, A. Devendran, Drag on a permeable cylinder in steady flow at moderate Reynolds numbers, Chemical Engineering Science 53 (16) (1998) 2859-2869. doi:http://dx.doi.org/10.1016/S0009-2509(98)00117-1. URL http://www.sciencedirect.com/science/article/pii/S0009250998001171

[23] M. Vanni, Creeping flow over spherical permeable aggregates, Chemical Engineering Science $55 \quad$ (3) (2000) 685-698. doi:http://dx.doi.org/10.1016/S0009-2509(99)00316-4. URL http://www.sciencedirect.com/science/article/pii/S0009250999003164

[24] P. Vainshtein, M. Shapiro, C. Gutfinger, Creeping flow past and within a permeable spheroid, International Journal of Multiphase Flow 28 (12) (2002) 1945-1963. doi:http://dx.doi.org/10.1016/S0301-9322(02)00106-4 URL http://www.sciencedirect.com/science/article/pii/S0301932202001064

[25] P. Vainshtein, M. Shapiro, C. Gutfinger, Mobility of permeable aggregates: effects of shape and porosity, Journal of Aerosol Science 35 (3) (2004) 383-404. doi:http://dx.doi.org/10.1016/j.jaerosci.2003.09.004. URL http://www.sciencedirect.com/science/article/pii/S0021850203004397

[26] C. Beckermann, R. Viskanta, Double-diffusive convection during dendritic solidification of a binary mixture, $\mathrm{PCH}$. Physicochemical hydrodynamics 10 (2) (1988) 195-213.

URL https://www . scopus . com/inward/record. uri?eid=2-s2.0-0023856142\&partnerID=40\&md5=98c 
[27] K. Vafai, S. J. Kim, Forced convection in a channel filled with a porous medium: An exact solution, ASME Journal of Heat Transfer 111 (4) (1989) $1103-1106$.

[28] A. J. Basu, A. Khalili, Computation of flow through a fluid-sediment interface in a benthic chamber, Physics of Fluids 11 (6) (1999) 1395-1405. arXiv:http://dx.doi.org/10.1063/1.870004, doi:10.1063/1.870004, URL http://dx.doi.org/10.1063/1.870004

475 [29] S. Bhattacharyya, S. Dhinakaran, A. Khalili, Fluid motion around and through a porous cylinder, Chemical Engineering Science 61 (13) (2006) 4451-4461. doi:http://dx.doi.org/10.1016/j.ces.2006.02.012. URL http://www.sciencedirect.com/science/article/pii/S0009250906001175

[30] S. Dunnett, C. Clement, A numerical study of the effects of loading from diffusive deposition on the efficiency of fibrous filters, Journal of Aerosol Science 37 (9) (2006) 1116-1139. doi:http://dx.doi.org/10.1016/j.jaerosci.2005.08.001. URL http://www.sciencedirect.com/science/article/pii/S0021850205001679

[31] R. Mardanov, S. Dunnett, S. Zaripov, Modeling of fluid flow in periodic cell with porous cylinder using a boundary element method, Engineering Analysis with Boundary Elements 68 (2016) 54-62. doi:http://dx.doi.org/10.1016/j.enganabound.2016.03.015 URL http://www.sciencedirect.com/science/article/pii/S0955799716300492

[32] M. Kelmanson, An integral equation method for the solution of singular 490 slow flow problems, Journal of Computational Physics 51 (1) (1983) 139-158. doi:http://dx.doi.org/10.1016/0021-9991(83)90084-0 URL http://www.sciencedirect.com/science/article/pii/0021999183900840

[33] M. Jaswon, G. Symm, Integral equation methods in potential theory and electrostatics, Academic Press, New York, 1977. 
[34] E. O. Tuck, Calculation of unsteady flows due to small motions of cylinders in a viscous fluid, Journal of Engineering Mathematics 3 (1) (1969) 29-44. doi:10.1007/BF01540828.

URL http://dx .doi .org/10.1007/BF01540828

[35] M. Abramowitz, I. Stegun, Handbook of Mathematical Functions: With Formulas, Graphs, and Mathematical Tables, Applied mathematics series, Dover Publications, 1964.

[36] R. F. Mardanov, O. V. Soloveva, S. K. Zaripov, Flow past a porous cylinder in a rectangular periodic cell: Brinkman and Darcy models comparison, IOP Conference Series: Materials Science and Engineering 158 (1) (2016) 012065 .

URL http://stacks . iop.org/1757-899X/158/i=1/a=012065

[37] Y. Kim, D. W. Kim, S. Jun, J. H. Lee, Meshfree point collocation method for the stream-rorticity formulation of $2 \mathrm{~d}$ incompressible navierstokes equations, Computer Methods in Applied Mechanics and Engineering 196 (33-34) (2007) 3095-3109. doi:http://dx.doi.org/10.1016/j.cma.2007.01.018. URL http://www.sciencedirect.com/science/article/pii/S0045782507000734 